\title{
Difficulté de vivre avec « le passé devant soi »: ne pas tomber dans le réalisme ordinaire chez Gilbert Gatore
}

\author{
Valérie Dusaillant-Fernandes \\ Université de Waterloo
}

Gatore a le projet, dès 2007, d'écrire une trilogie intitulée Figures de la vie impossible, dans laquelle il désire revisiter le génocide des Tutsi au Rwanda par le biais de la fiction, avec une question-phare qui guide sa démarche, qu'il révèle à Nathalie Colleville dans un entretien qu'il lui a accordé en 2010: "Comment envisager le présent et le futur lorsque l'on a un passé que l'on ne parvient pas à dépasser, un passé qui ne reste pas derrière soi ? " Justement, le passé de Gatore le rattrape chaque fois qu'il avance vers de nouveaux horizons, vers de 
nouveaux défis. En 1994, la famille Gatore fuit le génocide ; Gilbert a alors 13 ans. Les Gatore errent pendant quelques années dans les pays voisins, puis passent la frontière pour aller vers la République démocratique du Congo. Arrivés en Europe, les parents s'établissent définitivement en France en 1997. Une dizaine d'année plus tard, Gilbert Gatore fait son entrée sur la scène littéraire avec la parution de son premier roman, Le Passé devant soi, qui est aussitôt reconnu par la critique et le public ${ }^{1}$.

Dans cet article, il s'agira d'étudier, dans un premier temps, la structure narrative du récit, qui montre cette résistance à ne pas tomber dans le réalisme des témoignages. En nous basant sur les recherches de Justin Bisanswa et de Josias Semujanga sur le roman africain, nous examinerons l'entrecroisement bien calculé de deux récits - le premier, une sorte de fable fragmentée sur Niko le paria, constitué de courts paragraphes numérotés; le second, un récit sur Isaro à la temporalité perturbée - et l'importance d'une voix-off qui accompagne le lecteur dans son cheminement à découvrir la nature humaine. Dans un deuxième temps, nous serons amenée à nous arrêter sur le destin singulier des deux protagonistes pour qui le Rwanda est synonyme de souffrance et d'exclusion. Les apports théoriques freudiens nous aideront également à nous pencher sur la fonction de l'angoisse de la solitude et la peur d'affronter un passé ou une vérité qui dérange.

1 La même année, l'auteur est le lauréat du Prix Ouest-France Étonnants Voyageurs, prix décerné par de jeunes lecteurs (15-20 ans) passionnés de lecture. 


\section{Choix de la fiction et structure du récit}

La parution du Passé devant soi s'inscrit dans l'étape importante du travail de mémoire sur le génocide contre les Tutsi au Rwanda dans la société française amorcée en 1998 avec une opération lancée par l'Association Arts et Medias d'Afrique (A.M.A.). Cette année-là, les deux fondateurs, Nocky Djedanoum et Maïmouna Coulibaly, ont organisé un festival de littérature au Rwanda et ont conçu un projet qui consistait à regrouper des artistes africains pour réfléchir sur la tragédie rwandaise et en rendre compte par l'écriture ${ }^{2}$. Le festival Fest'Africa de 1998, intitulé «Rwanda: écrire par devoir de mémoire», a permis ultérieurement la publication de nombreuses œuvres littéraires ou artistiques. Deux ans plus tard, en 2000, toujours en France, c'est le travail du reporter malgache Jean Hatzfeld qui a retenu l'attention des lecteurs avec son triptyque littéraire regroupant des témoignages de victimes et de bourreaux du génocide 3 . Plus récemment, il faut souligner la publication de textes autobiographiques ou fictionnels d'auteurs rwandais installés

2 Rappelons que ce sont deux journalistes africains, le Tchadien Nocky Djedanoum et l'Ivoirienne Maïmouna Coulibaly, qui ont fondé l'Association Arts et Médias d'Afrique (A.M.A) en 1992 à Lille, car ils avaient observé que les Français ne prêtaient aucune attention à la littérature africaine. Les principaux participants de cet événement artistique étaient les écrivains Nocky Djedanoum (Tchad), Boubacar Boris Diop (Sénégal), Koulsy Lamko (Tchad), Tierno Monemembo (Guinée), Monique Llboudo (Burkina-Faso), Véronique Tadjo (Côte d'Ivoire), Abdourahman Waberi (Djibouti), Meja Mwangi (Kenya), Jean-Marie Vianney Rurangwa et Vénuste Kayimahe (Rwanda) ; Un sculpteur, Bruce Clark (Afrique du Sud), et un cinéaste, Samba Félix N'Diaye (Sénégal), se sont joints aussi à ce groupe. Chaque année, l'association continue d'organiser un festival de littérature africaine qui favorise la diffusion de la culture africaine dans la région de Lille en France.

${ }^{3}$ Dans le nu de la vie (Paris, Seuil, 2000), Une saison de machettes (Paris, Seuil, 2003) et La Stratégie des antilopes (Paris, Seuil, 2007). 
en France comme Scholastique Mukasonga, Benjamin Sehene ${ }^{4}$ et Gilbert Gatore. Si nous insistons sur les textes de ces trois auteurs, c'est particulièrement à cause de leur jeu avec la fiction pour raconter les atrocités des massacres de 1994. Par exemple, c'est grâce à la forme de la nouvelle ou du roman que Mukasonga déploie les variations imaginaires de ses souvenirs d'enfance et expulse dans le champ public les angoisses qui la hantent. Nous sommes consciente aussi que la démarche de ces auteurs suit celle d'écrivains comme Tierno Monemembo qui, comme le souligne Josias Semujanga dans Le Génocide, sujet de fiction?, s'attache par la fiction à « contourner le génocide pour mieux le cerner » (p. 91).

Bien loin des témoignages, des essais et des autofictions, Le Passé devant soi est un de ces romans qui interpellent par sa construction textuelle, qui peut être déroutante pour certains lecteurs. En effet, en conviant le lecteur vers l'indicible de la peur et de l'angoisse et vers la quête de la difficulté de vivre avec « le passé devant soi », Gatore nous conduit au cœur d'une réflexion sur les séquelles laissées par l'horreur génocidaire. L'auteur a réussi son pari, celui d'amener le lecteur à s'interroger sur le destin tragique de ses deux protagonistes, Niko-le-singe et Isaro Gervais, deux êtres victimes d'un génocide dans un pays qui reste sans nom dans le roman. En effet, le Rwanda apparaît par des allusions telles que « là où ils se sont massacrés, il y a quelques années» (p. 28), «la route montait la colline du sein-de Mukaneza (chaque colline était supposée représenter le sein d'une beauté légendaire » (p. 144),

\footnotetext{
4 Mukasonga a publié quatre ouvrages à ce jour : Inyenzi ou les cafards (2006), La Femme aux pieds nus (2008), L'Iguifou. Nouvelles rwandaises (2010) et Notre-Dame du Nil (2012). Sehene est l'auteur du roman Le Feu sous la soutane (2005).
} 
les notations indirectes comme le fait de mentionner les massacres avec des machettes ou encore tous les prénoms de personnages en kinyarwanda 5 . Le but de Gatore était d'offrir une portée universelle, une réflexion sur tous les génocides.

Gatore est un de ces écrivains qui utilisent l'écriture comme «instrument d'exploration du réel, de figuration de l'Histoire, d'analyse de la société » (Bisanswa, p. 12). Texte atypique, Le Passé devant soi s'impose comme un roman qui se tient à l'écart du « réalisme ordinaire » qui, selon Bisanswa, est voué "à une platitude ou à un artifice qui sont négation de l'art» (p. 16). Bisanswa remarque que le réalisme "s'exprime pleinement par une écriture qui se trouve en plus être retorse et dissimulée. Il relève de toute sorte de conventions expressives » (p. 15). C'est ainsi que nous percevons le texte de Gatore. Un roman qui, pour dire le réel, se construit par des dispositifs fictionnels, des stratégies discursives et narratives dans lesquels l'auteur développe le thème du génocide. L'écrivain rwandais, dans les traces de Tierno Monemembo, « cherche des formes susceptibles de représenter l'horreur de l'extermination afin de provoquer la réflexion du lecteur sur la portée morale de la fiction du génocide par des mots justes", pour reprendre les termes de Josias Semujanga (p. 23).

Ce qui frappe au premier abord, c'est la figure de l'apostrophe, qui ponctue le texte d'un bout à l'autre. Au début du roman, cette voix-off, que l'on croit être celle du narrateurauteur, met en garde "l'inconnu » qui va s'aventurer à lire le roman que le «voyage [lui] sera peut-être insoutenable»

5 Nicki Hitchcott (2013) explique, entre autres, pourquoi Gatore omet volontairement de nommer le Rwanda dans son roman, tirant la conclusion que le texte suggère une tension entre le souvenir et l'oubli. 
(p.11). Quelques pages plus loin, la voix avertit de nouveau le " curieux » auquel elle s'adresse que, ces pages passées, il ne lui sera plus possible de "partir aussi facilement qu'[il] pourr[ait] le faire maintenant» (p.15). Tel un conteur, le narrateur s'impose pour protéger le lecteur d'images et de passages difficiles (p.69), pour retenir son attention sur un trait particulier d'un des personnages (p.60), pour lui demander de laisser libre son imagination (p.104) ou encore pour le soumettre à la réflexion. En fait, ce n'est qu'à la fin du roman que l'on apprend, par une mise en abyme, que la voix de ce récit est celle d'Isaro Gervais, qui vient de raconter le destin extraordinaire du personnage principal de son récit qui n'est autre que Niko. Ainsi, Gatore se sert du lecteur comme d'un élément de base de la structure romanesque de son ouvrage. Le destinataire n'est plus qu'un acteur du récit en cours, celui élaboré par Isaro. Dès lors, l'apostrophe favorise des digressions où le lecteur réel est invité à s'identifier au « lecteur implicite », comme le remarque Wolfgang Iser :

Le concept du lecteur implicite [...] est une structure textuelle préfigurant la présence d'un récepteur sans nécessairement le définir : ce concept préstructure le rôle à assumer par chaque récepteur, et ceci reste vrai même quand les textes semblent ignorer leur récepteur potentiel ou l'exclure activement. Ainsi, le concept du lecteur implicite désigne un réseau de structures invitant une réponse, qui contraignent le lecteur à saisir le texte. » (p. 34)

Par cette stratégie textuelle, Gatore alerte non seulement le lecteur et le contraint à réagir sur la lecture en cours, mais affirme aussi son rôle omnipotent en tant que créateur de l'œuvre. Cela dit, si la forme interpellatoire prend celle d'une adresse directe d'Isaro au lecteur implicite, il faut quand même souligner cette présence auctoriale dans le texte par des 
épiphrases du narrateur-auteur qui sont glissées par deux fois dans Le Passé devant soi sans guillemets, indiquant qu'il ne s'agit pas d'une adresse au lecteur implicite.

En effet, le lecteur réel est directement interpellé lorsque le narrateur-auteur utilise la deuxième personne du pluriel et l'impératif pour solliciter son imagination :

Imaginez la belle à la façon qui vous convient. Si cela vous aide à être avec elle, représentez-vous assis derrière ou à côté d'elle, partageant sa vue sur l'extérieur et sur elle-même. Dans tous les cas, elle ne peut pas vous voir. Si cela vous paraît préférable, vous êtes en elle, vous êtes ses yeux, son souffle ou son souvenir. » (p. 89)

En invitant le lecteur à se placer aux côtés d'Isaro, Gatore lui propose d'entreprendre le même travail de réflexion que son personnage écrivain et brouille les limites en lui proposant même de devenir Isaro. Le lecteur réel va alors devenir le témoin intime de la tentative de reconstruction puis de la chute mortelle d'Isaro. Vers la fin du roman, le narrateur-auteur apostrophe à nouveau le lecteur réel, lui rappelant sa place de coparticipant resté « dans l'ombre » d'où il l'observe « depuis le début» (p. 183). Cependant, le lecteur réel doit bien se garder de tomber dans le piège de sombrer dans le désarroi d'Isaro dans la quête de ses origines. Par un jeu de narration et de mise en abyme, le lecteur est inséré dans la trame narrative complexe dans le simple but d'être le témoin et le passeur d'une histoire qui ouvre le débat sur la résurgence du passé dans le présent. Si dans certains cas, comme l'indique Josias Semujanga, «le passé devient principe d'action pour le présent, pour la quête de la justice par exemple» (p. 284), il représente également le lieu de l'échec par cette impossibilité à fuir les souvenirs. Peut-on dépasser ses peurs ou ses angoisses face à 
l'événement traumatique ou domestiquer la douleur et vivre avec? En lisant les destins de Niko et Isaro, il semble que la tentative de refoulement ou d'oubli du trauma vécu est un échec pour les deux protagonistes.

\section{Niko : entre empathie et dégoût}

«Éternellement puni », honteux et coupable, Niko est devant une impasse : il ne peut plus affronter le souvenir de ceux qu'il a massacrés et qui errent dans son esprit. C'est au moyen de 251 fragments numérotés dans treize chapitres que l'on découvre la personnalité et le parcours de Niko. Pourquoi cette numérotation? En fait, elle correspond à « un mode d'écriture » qui, selon l'auteur lui-même, « donne la place aux pensées, mais dans le même désordre que celui dans lequel elles peuvent surgir » $\left(2011,10^{\prime} 03\right)^{6}$. Autrement dit, la numérotation autorise le désordre, c'est-à-dire de passer d'une idée ou d'une réflexion à une autre, et permet aussi de traduire les troubles psychologiques dont souffre Niko.

Décrit au départ comme un homme naïf, perdu et abandonné de tous, auquel le lecteur s'attache, le personnage de Niko se révèle être un être monstrueux au passé sanguinaire. Même au premier abord, si son physique paraît harmonieux, c'est quand il dévoile ses dents "immenses», "miteuses» et « désordonnées », qu'il ressemble à un singe, voire un démon (p. 98). Gatore a créé un personnage engagé dans des tueries abominables, mais foncièrement humain dans son parcours, ses

6 Gatore admet aussi avoir été inspiré par deux autres auteurs qui ont pratiqué ce type de numérotation dans leur texte: Alain Robbe-Grillet dans La Reprise et John Maxwell Coetzee dans Au cour de ce pays (10'58'). 
peurs et ses angoisses. La construction narrative et descriptive du personnage de Niko a un double objectif: décrire les barbaries génocidaires dans toute leur trivialité, mais aussi restituer la dimension humaine à un conflit qui a détruit le lien social et conditionné l'esprit de milliers d'individus dans un but destructeur. Dès lors, en lisant Le Passé devant soi, le lecteur peut être amené à ressentir des émotions conflictuelles en parcourant les pages du récit, oscillant entre l'empathie pour Niko enfant et le dégoût pour le meurtrier passif à l'égard de sa propre barbarie.

Il faut dire que Niko le bourreau se pose aussi comme victime d'une déshumanisation, voire d'une chosification qui le rend vulnérable à la manipulation ou à l'endoctrinement dès son plus jeune âge. Ainsi, le jour de sa naissance, sa mère ne se rend même pas compte qu'elle accouche de lui. Le petit corps « roul[e] sur le sol sans que personne le remarqu[e]» (p. 79) et c'est finalement le chien de la maison qui « avertit la présence d'un nouvel être» (p. 80). Dans ce passage sur la naissance de Niko, le substantif « chose » est employé deux fois, soulignant le fait que le processus de déshumanisation est déjà en cours. Si certains croient que Niko est muet parce que traumatisé par la mort en couches de sa mère, il reste cependant un incompris pour les autres : beaucoup croient qu'il joue un jeu et il devient très vite la proie de toutes sortes de railleries et de punitions injustes. Sa grand-mère a même l'impression de tenir dans ses mains « une horrible poupée possédant tout d'un être humain sauf la voix » (p. 80). Incompris, délaissé par ses parents qui ne voient en lui qu'un fardeau, Niko n'existe aux yeux de tous que par son prénom qui, en kinyarwanda, ne signifie rien d'autre que «hé! » ou bien encore «toi là-bas» (p. 81). Il fait donc partie des anonymes, de ceux qui n'existent pas ou si peu, 
comme l'est d'ailleurs le personnage d'Isaro. À ce propos, Catherine Coquio constate que la fiction permet un «lieu de partage imaginaire entre la rescapée orpheline et le tueur coupable» (2010, p.244). En effet, le destin de Niko est contrôlé par Isaro, qui décide de faire de lui un être fragile au départ. Telle la jeune orpheline, Niko est d'emblée symboliquement «abandonné » par les siens, puisque jeune, il est atteint par le mal au plus profond de son être. Cette fragilité le condamne à vivre perpétuellement dans la peur.

Face aux images insoutenables, Niko est « occupé à avoir peur» (p. 128), comme si la peur était devenue une tâche physique, comme travailler à sa forge, une activité quotidienne à laquelle il ne peut malheureusement pas échapper et dont il doit s'acquitter. Son travail n'est plus de fondre de l'acier pour créer des objets utiles, mais «d'avoir peur». Il faut dire que l'angoisse ou la peur ne l'a jamais quitté. Rappelons que ces deux mécanismes intra-psychiques sont quelque peu différents : d'un point de vue psychanalytique, l'angoisse est un trouble anxieux sans objet réel externe identifié ; on peut être angoissé sans représentation directe que ce qui nous rend mal à l'aise. En revanche, une émotion comme la peur est immédiate et se rattache à un objet, une personne ou une situation extérieure à nous-mêmes ; on réagit subitement à cet objet pour se protéger. Depuis sa naissance, Niko a vécu angoissé face au traumatisme de sa naissance et au rejet des membres de sa communauté. Ce jour-là, devant ce groupe d'agresseurs armé et menaçant qui le défie de tuer un homme qui ressemble à son père, c'est la frayeur qui le tenaille. Niko n'a pas été préparé au danger qui existe devant lui et, donc, il doit gérer rapidement l'émotion qui le submerge. 
En réaction, se pose l'acte de résistance face à ces exécutions gratuites et barbares auxquelles Niko doit participer pour la première fois. Dans le fragment 164, le lecteur est plongé dans les pensées du muet, qui lui permettent d'évaluer son état émotionnel :

«Au suivant!» hurle-t-on. «Toi, là-bas!» Niko fait semblant d'hésiter, de ne pas entendre que c'est à lui qu'on parle, puis se rappelle ce que cela peut lui coûter. Alors, il fait les quelques pas qui doivent lui permettre d'avoir la victime à sa portée. Pendant ce laps de temps, toutes sortes d'idées lui traversent la tête : fuir et recevoir une balle dans le dos, feindre un malaise et recevoir une balle dans la poitrine, expliquer qu'il ne peut pas faire ça et recevoir une balle entre les yeux. Dans tous les cas, après l'avoir abattu, on tuerait celui ou celle qui doit à l'évidence mourir, on jetterait leurs deux cadavres par-dessus les autres et crierait : «Au suivant !» (p. 129)

Tous les cas de figure envisagés semblent ramener Niko à une mort certaine s'il n'obtempère pas aux ordres. Pour autant, Niko ne semble pas prêt à mourir puisqu'entre l'instinct de vie et l'instinct de mort qui pourrait le sauver du fardeau de son existence, il choisit de vivre et donc de commettre l'irréparable. Freud explique cela dans son chapitre "Considérations actuelles sur la guerre et sur la mort » d'Essais de psychanalyse :

Le fait est qu'il nous est absolument impossible de nous représenter notre propre mort, et toutes les fois que nous l'essayons, nous nous apercevons que nous y assistons en spectateurs. C'est pourquoi l'école psychanalytique a pu déclarer qu'au fond personne ne croit à sa propre mort ou, ce qui revient au même, dans son inconscient chacun est persuadé de sa propre immortalité. (p. 19)

La notion de voir sa mort comme spectateur paraît convenir au personnage de Niko. Il s'imagine mourir et envisage différents scénarios, mais ne tient pas à aller jusqu'au bout, persuadé que 
tout cela serait peine perdue puisque l'on finirait par tuer l'autre personne. De plus, selon Freud, «à en juger par nos désirs et souhaits inconscients, nous ne sommes nous-mêmes qu'une bande d'assassins » (p. 25), car nous préférons la mort de l'autre, de l'étranger, plutôt que notre propre mort. En somme, nous serions tous des lâches face à la mort.

Dans le fragment 165, c'est par des questions rhétoriques que l'auteur amène le lecteur à réfléchir sur le choix de Niko :

Que faut-il faire lorsque la résistance, même par le sacrifice de soi, ne permet de sauver rien ni personne ? Est-ce que la main de celui qui tue ainsi a d'autres motifs de tuer que celui de préserver? Pourquoi, en ce moment, Niko est-il incapable de faire ce qu'il était sûr de faire quelques minutes auparavant? (p. 129)

L'auteur n'attend pas de réponse, mais une réflexion sur l'agir de l'autre et ses conséquences. Avant de juger Niko le bourreau, ne doit-on pas juger d'abord ses agresseurs, qui l'ont conduit à faire ce choix impossible? «L'instinct de survie justifie-t-il qu'on tue » son prochain (p.132) ? Mais, rappelons-nous, Niko n'a jamais été humain. "Chose » depuis sa naissance, il s'avère insensible à la mort des autres et finit par trouver une communauté qui l'accepte comme il est, même avec son mutisme, qui « contribue à imposer son autorité » (p. 134). Niko n'est plus qu'une machine à tuer.

\section{De victime au bourreau puni : rêves et cauchemars}

Alors, une fois que la tuerie est finie, que se passe-t-il ? La vie reprend son cours et personne ne parle de ce qui s'est passé. Toutefois, toute tentative d'effacer le passé est vaine. Les images traumatiques reviennent hanter les nuits du bourreau. 
Assiégé de rêves et portant sans cesse le masque de la honte et la culpabilité, il s'exclut du monde des humains en se réfugiant dans une grotte «qui ne ressemble à rien » (p. 30), où il espère trouver ce fameux monstre qui va le dévorer et ainsi être « débarrassé de la faim, de la fatigue et surtout de la nausée qui le torturent» (p. 24). Longtemps refoulés, les souvenirs des massacres commis empêchent Niko de vivre. Freud a justement observé que, lorsqu'un individu a été soumis à un événement traumatique, son appareil psychique met en mouvement des moyens de défense de refoulement qui protègent son inconscient (1920, p.18). Selon Freud, le rêve est l'un des moyens d'exploration de l'inconscient. Toutefois, avec le rêve traumatique, le sujet est fixé au trauma vécu et il se voit ramené sans cesse aux souvenirs. Il constate aussi que les rêves de ses malades se «composent de tableaux remontant à l'époque où ils étaient bien portants ou se rattachant à leur espoir de guérison» (1920, p.13). Or, un des rêves de Niko commence plutôt bien et décrit cette journée où il se promène de «bonne humeur» avec son père sous un "soleil radieux» (Gatore, p.32) dans une forêt composée d'eucalyptus, de bananiers, d'hibiscus et d'oiseaux. Les deux hommes, que «rien n'inquièt[e]», semblent profiter de ce moment de paix pour "gambader» et apprécier le silence à travers le feuillage (p. 32). Précisons que cette même scène revient quelques pages plus loin avec, cette fois, la présence d'une femme inconnue (p. 58) montrant l'importance de l'inconscient à se retrancher dans un état de plaisir pour éviter l'angoisse. Cela dit, la compulsion de répétition à revivre le trauma fait irruption dans le rêve et se traduit par un changement de décor, avec notamment l'apparition de corbeaux, d'une «lumière rouge, sombre et visqueuse » (p. 32-33). Les arbres autour du père et 
du fils s'anthropomorphisent en «une armée de géants immobiles» (p.33). La frayeur fait alors place au bonheur7. Comme un monstre sorti de l'enfer, Niko crache du feu aux alentours, brûlant son père qui lui demande, " avant de tomber étouffé par la fumée: "pourquoi mon fils ?"» (p. 33). MarieOdile Godard, dans Rêves et traumatismes ou la longue nuit des rescapés, note que les rêves traumatiques apparaissent parce que «le pare-excitation a été défaillant» et que "les images sont restées en suspens, prêtes à revenir sous forme d'hallucinations » (p. 50). Au demeurant, les hallucinations ne sont pas les seuls symptômes de la réminiscence des souvenirs traumatiques. Niko est pris de tremblements (p. 37), de convulsions (p.38 et 148), de vomissements (p.39 et 143), d'évanouissements (p. 124).

Les mots du père, dans l'hallucination de Niko, ne sont pas anodins. Dans la culture et la croyance rwandaises, il ne faut pas oublier qu'Imana est au centre de la religion traditionnelle. Ce dieu tout-puissant protège et habite chaque être humain, chaque animal ou chaque objet sur terre. De plus, en tant que grand maître absolu, Imana est le chef de file des autres esprits comme les Imandwa et les Abazimu; ce sont ces derniers qui peuvent être dangereux. Marie-Odile Godard explique que

plus les Abazimu sont proches dans la lignée directe d'une personne, plus ils lui sont favorables. Plus ces Abazimu appartiennent à des lignées éloignées, plus ils sont dangereux. Le Guterekera est une cérémonie préventive pour satisfaire, apaiser «ses» Abazimu. Si tout est fait selon la tradition, les Abazimu seront protecteurs des vivants. Dans le cas contraire, leur colère peut provoquer des maladies mentales, des cauchemars ou autres manifestations négatives. (p. 115-116)

7 À ce propos, Freud affirme que la frayeur est un état auquel une personne n'est pas préparée ; il y a donc un effet de surprise (1920, p. 13). 
Nous pensons que Niko n'a pas eu le temps d'apaiser les esprits des défunts en pratiquant le Guterekera et que, de ce fait, il est devenu la proie des esprits vengeurs. Les singes qui habitent avec lui dans la grotte ne sont que le produit de son imagination, la représentation animale des Abazimu8. D'ailleurs, dans le chapitre 3 , un des singes est sauvagement abattu d'une balle qui était destinée à Niko. Celui-ci, qui voit ce singe comme son « ange gardien » (p. 47), se demande justement si ces singes enterrent leurs morts ou s'ils les laissent "pourrir là où ils s'écroulent» (p. 50). À bien des égards, Niko est touché par le comportement de ce singe, mais il ne procède pas à son inhumation sur le terrain familial comme le veut la tradition rwandaise. Au contraire, il décide de le vider et de l'accrocher à une voûte, pensant que l'esprit du singe le protégera. Cependant, l'esprit du défunt non enterré est un esprit errant et, de ce fait, Niko empêche l'esprit du singe de reposer éternellement. Du reste, d'autres singes apparaissent avec, dans les yeux, une «supplication» (p. 62). Par leurs regards durs et froids, ils exigent qu'un dernier hommage soit rendu à l'un des leurs (p. 62).

Tout bien considéré, les animaux sont une mise en miroir des émotions des hommes. Effectivement, le texte de Gatore se caractérise par un regard autre sur l'immoralité (corps laissés sur place ou exposés, dignité humaine déniée), le sacrilège (inhumation non respectée) et la violence gratuite en dénonçant l'idéologie destructrice du génocide par une allégorie. Dans ces conditions, quelle est la moralité de l'histoire de Niko ? Niko est devenu impuissant face à son imagination,

\footnotetext{
8 Rappelons-nous qu'étant enfant, Niko avait été surnommé « Niko-le-singe », certainement en raison de la croyance villageoise qui pensait qu'à cause de son mutisme, le jeune enfant ne pouvait ni penser, ni rêver (Gatore, p. 35).
} 
aux esprits, aux souvenirs qui l'envahissent: "visages sur lesquels il a craché », urine sur les corps de ses victimes, viols, " corps jetés dans les fosses, brûlés, coulés, entassés, dissous » (p. 146). Alors, si les Abazimu sont les singes de la grotte, Niko est condamné à se sentir coupable jusqu'à sa mort. D'ailleurs, il comprend très vite qu'il est prisonnier de leurs regards pour l'éternité (p. 156) et qu'il doit assumer la responsabilité de ses actes et du contenu de ses rêves traumatiques. Voilà pourquoi, chaque fois qu'il ne peut plus faire face à tous ces cauchemars et qu'il essaie de s'éloigner de la vue des singes, le chef du groupe le sanctionne sévèrement, le saisissant par le cou, le secouant violemment, le transperçant « d'un regard noir » et laissant sur le sol son pauvre «squelette inconscient» (p. 156). Niko entre en totale dépendance des autres et, au moindre écart, la «punition tombe, brutale, implacable» (p. 156). Les nombreuses humiliations imposées par les singes l'amènent à se poser des questions sur son sort et, dans le fragment 212, le lecteur est plongé dans les interrogations qui déferlent dans sa tête : «A-t-il essayé d'échapper à la justice? De se punir? Une punition à la hauteur de sa culpabilité existe-t-elle? La mort est-elle une solution? Et l'isolement? Les singes exécutent-ils son châtiment? En ont-ils reçu la mission ? De qui ?» (p. 159) Complètement soumis, Niko est certainement la proie des Abazimu, ces mauvais esprits, puisque dans les yeux des singes, il voit Uwitonze, son maître d'école qui a tenté, au péril de sa vie, de protéger des Tutsi, Uwera, la vendeuse de vin de banane sauvagement violée, et enfin Shema, le conteur du village, forcé de quitter les lieux de peur "qu'il évoquât quelque chose qu'il valait mieux taire» (p. 171). Exclu, condamné à souffrir, Niko observe qu'«Uwitonze, Uwera et Shema, compagnons en pensée ou créations de son imagination»(p.172), ne sont là 
que pour mourir et emporter Niko avec eux. Petit à petit, les protecteurs d'un temps deviennent des persécuteurs de l'obscurité. Ce qu'Uwitonze, Uwera et Shema n'ont pas pu faire avant, ceux contre qui ils essayaient de se protéger, les meurtriers, ils les affrontent à l'heure de leur mort. Mais rappelons que Niko sort de l'imagination d'Isaro et que c'est donc elle qui l'astreint à une fin peu enviable.

Amadouer les Abazimu serait peine perdue. Sentant la fin venir, Niko ne peut plus résister à leurs regards et se trouve ainsi «inondé par la douleur qui l'étouffe» (p. 178). Les derniers fragments sur Niko racontent ses derniers instants de vie et proposent, selon nous, la morale de la fable. $\mathrm{Ne}$ supportant plus cette vie de reclus et ce sentiment d'être "puni pour n'avoir pas su utiliser une vie qu'il n'avait pas désirée », Niko choisit l'enfer puisqu'il «ne se sent pas autorisé à penser au paradis» (p. 179). Mieux vaut une fin partagée avec les démons que le regard éternel des victimes. Ainsi, la morale implicite de la fable de Gatore serait, pour reprendre celle d'une de Jean de La Fontaine, Le Lion et le rat, "Patience et longueur de temps font plus que force ni que rage ». Cela dit, ce que nous privilégions, c'est l'image de la patience pour trouver justice et apaisement. Si la loi du plus fort n'est pas la meilleure, celle du temps associé à la patience est incontestablement la plus sereine et la plus sûre. Niko a toujours gardé depuis sa naissance un noyau de souffrance au plus profond de lui-même, mais cette douleur interne qui s'est extériorisée par des massacres a eu gain de cause au fil du temps, comme le souligne le fragment 248: "Impasse et douleur. Heureusement, il meurt. » (p. 179) L'usage de l'adverbe «heureusement» a un double sens : il peut signifier que la mort est un soulagement face aux épreuves ou, au contraire, une juste punition 
répondant aux délits commis, punition qui lui est infligée par Isaro, sa créatrice, celle qui décide de son sort. Ainsi, tout comme Niko, à qui on a dénié toute existence dès sa naissance, le personnage d'Isaro, jeune fille adoptée, va découvrir que ses parents lui ont caché ses origines rwandaises, rejetant volontairement son passé quelque peu lourd à porter.

\section{Isaro l'adoptée : d'un retour à l'autre}

Gatore a subtilement placé l'histoire d'Isaro Gervais dans les treize chapitres qui suivent les fragments sur Niko. Ces chapitres consacrés à cette jeune étudiante qui vit à Paris dévoilent un autre aspect des conséquences du génocide : les enfants orphelins. Rescapés, survivants, qu'est-ce qu'il est advenu de ces enfants laissés pour compte ${ }^{9}$ ? Certains ont été recueillis par des proches, d'autres placés dans des orphelinats et quelques-uns sauvés par des anonymes. Comme pour bon nombre d'enfants rwandais, l'enfance d'Isaro a été marquée à jamais par les indicibles violences perpétrées par des barbares avides de sang. Toutefois, au début du roman, le lecteur se rend compte qu'Isaro ne connaît pas ce passé traumatique. À l'instar de Niko, qui vit dans l'obscurité dans sa grotte, Isaro habite dans son petit appartement sombre (p. 11, 24 et 40), où elle s'est recluse pour écrire un livre qu'elle intitulera À la mémoire de...

En fait, ce qui a déclenché chez ce personnage l'acte d'écriture, c'est le retour dans son pays natal, une patrie aux

\footnotetext{
9 Dans un communiqué de presse provenant de l'UNICEF daté du 6 avril 2004, le nombre d'enfants orphelins à la fin du génocide de 1994 se porte à 95000 <lighttp://www.unicef.org/french/media/media_20325.html>.
} 
mille collines et aux mille souffrances qui lui était étrangère. Isaro s'est construite une vie ordonnée en France et son monde s'effondre quand elle est ramenée à une réalité qui la dépasse. Elle a toujours su qu'elle venait d'ailleurs, avec reconnaissance, et admet que ses parents adoptifs sont des «anges. Des sauveteurs et des gardiens providentiels dont les ailes [ont] permis à la petite perle qu'elle était, jetée hors de l'eau, de continuer à croître» (p. 33). Cependant, en dépit de l'amour qu'ils lui ont donné, Isaro a toujours voulu connaître les circonstances de son adoption. Désemparée, la jeune femme se lance non seulement dans une quête investigatrice d'un passé caché par les parents adoptifs, mais aussi dans la création d'un roman qu'elle offrira en «mémoire d'elle » (p. 183).

Le récit évolue en une suite de plusieurs analepses qui finissent par produire un désordre temporel. Malgré une certaine linéarité qui structure le récit et qui tend vers une fin à laquelle le lecteur ne s'attend pas, le déroulement chronologique déraille plusieurs fois, ce qui découpe certes le texte, mais favorise une meilleure compréhension de l'état psychologique d'Isaro face à son travail de recherche sur ses origines. Gatore manipule les événements de la vie de ses personnages en instaurant des allers et retours entre le présent, le passé et le futur.

D'autre part, ces glissements d'un temps à un autre participent à la fragmentation du récit, comme cela était le cas avec l'histoire de Niko. Les analepses complétives ${ }^{10}$ du roman

\footnotetext{
10 Genette définit l'analepse complétive - aussi appelée «renvoi »-comme tous « les segments rétrospectifs qui viennent combler après coup une lacune antérieure du récit, lequel s'organise ainsi par omissions provisoires et réparations plus ou moins tardives » (1972, p. 92).
} 
restituent les raisons du comportement d'Isaro. L'expression de son intériorité est accompagnée de retours en arrière permettant au lecteur de mieux cerner ses états d'âme et ses peurs face à la recherche entreprise. Ainsi, quand dans le prologue, on lit qu'elle est «immobile» (p.11 et 24), « concentrée» (p.11) et cloîtrée chez elle (p.12), c'est dans le chapitre premier que le recours à l'analepse évoque ce fameux matin où la nouvelle a tout "figé dans un coin de sa tête" (p. 25). Isaro prend soudainement conscience de « la lâcheté de sa routine» (p. 29) et qu'il est temps de rattraper le temps perdu en perçant le mystère de ses jeunes années. Le « comblement rétrospectif» (Genette, 1972, p. 93) ne s'effectue pas d'un seul coup, mais au fil des pages. D'autres analepses expliquent, par exemple, comment elle a toujours vécu avec ce «malaise » à l'intérieur d'elle-même, voulant se détacher de plus en plus de ses parents adoptifs et de son petit ami (p. 54 et 90), ou encore sa tentative de suicide pour «faire éclater aux yeux de tous son malheur, indiscutable et gênant» (p. 63). Ces divers retours en arrière dévoilent une jeune femme certes fragile, mais libre au milieu du «désert» qu'elle s'est créé autour d'elle, une fois arrivée à Paris.

Le retour au présent est marqué par de déictiques temporels comme «maintenant» (p.14, 52 et 53) et «aujourd'hui » (p.12 et 64) et ramène le lecteur au but que s'est fixé Isaro : retourner sur les lieux de son enfance, repartir dans son pays natal. C'est dans le présent qu'Isaro contrôle son destin et se donne les moyens d'aller jusqu'au bout du projet: enregistrer les témoignages des gens «autour de là où elle habitait » (p. 137), mais aussi et surtout réaliser des entretiens avec les massacreurs enfermés dans les prisons du Rwanda. Gatore revient ici sur le retour des rescapés rwandais qui 
avaient fui leur pays pendant le génocide. En effet, avec le personnage d'Isaro, il aborde le terrible besoin de revenir sur les lieux des massacres pour comprendre cette folie humaine ${ }^{11}$ qui a touché un pays. En dépit des témoignages, parfois difficiles, Isaro trouve l'amour en la personne de Kizito, qui lui apporte un soutien inconditionnel. À ses côtés, elle réapprend à être Rwandaise, à se reconstruire dans sa langue et dans sa culture.

C'est au détour d'une lettre du père adoptif envoyée à sa fille dans son pays natal que le véritable secret qui pèse sur les épaules d'Isaro est révélé. Cette lettre établit le fait que les parents adoptifs connaissaient les parents biologiques depuis six ans puisque tous travaillaient dans le lycée international de la ville, qu'ils étaient voisins et que, « lorsque les massacres ont commencé, ils sont venus se cacher chez [eux]» (p.153). L'auteur qui, par l'intermédiaire de la voix-off dans les formes interpellatoires, a mis en garde le lecteur depuis le début de son roman, continue cette stratégie dans la lettre même des parents, puisqu'on peut y lire ceci : «Il m'est aussi pénible de raconter ce qui suit que, j'imagine, pour toi de le lire.» (p. 154) Ce petit avertissement s'adresse à celui ou à celle qui lit lecteur réel et implicite - les lignes qui vont rapporter les faits tels qu'ils se sont passés. Cette phrase est aussi destinée, selon nous, à exciter la curiosité du lecteur, qui va enfin apprendre le contexte dans lequel Isaro a échappé à la mort. Le passage de la

\footnotetext{
11 Pour d'autres Rwandais, revenir au pays a consisté à retrouver les morts pour les enterrer dignement. L'écrivaine Scholastique Mukasonga est effectivement, après plusieurs années passées en France, retournée dans son pays d'origine pour tenter de trouver où reposent les siens. De même, Esther Mujawayo, est partie au Rwanda en 2005, en « espérant trouver des bribes de vérité, en espérant pouvoir récupérer enfin les dépouilles de [s]a sœur et de ses enfants » (p. 18).
} 
lettre révèle que, cachés sous un lit, les parents biologiques d'Isaro sont morts sous les balles et que sa sœur a été emportée par les miliciens. Par miracle, la petite fille a échappé au massacre bien que des balles auraient dû l'atteindre (p. 154). Gatore ne donne pas les détails, préférant tenir à distance la réelle brutalité des actes. D'ailleurs, tout le roman est construit de façon à rapporter les événements traumatiques sans pour autant tomber dans le sensationnel (discours de médias) ou dans le réel, comme on le trouve dans les récits de témoignages.

\section{La mort comme ultime délivrance}

Tout comme Niko, son personnage, Isaro vit l'exclusion. Certes, ses parents lui portent un amour "sans réserve ni condition » (p. 45), mais ils se sont obstinés trop longtemps à lui cacher son passé traumatique, niant ainsi son héritage identitaire et favorisant une remise en question de sa propre histoire personnelle. Dépossédée de ses racines par le secret, Isaro est doublement meurtrie quand elle retourne sur la terre de ses ancêtres. S'inscrire dans une généalogie quand tous les siens, proches et inconnus de la même ethnie, ont été rendus invisibles par un effacement collectif systématique en 1994, est du domaine de l'impossible. Gatore met en scène une jeune femme aux prises avec une expérience psychique effroyable vécue dans sa jeunesse et qui est toujours vécue sur le mode d'une rencontre avec la mort, la sienne mais aussi celle des autres. Cette période d'anéantissement est sans arrêt en action et la compulsion à la répétition amène Isaro à réactualiser les expériences déplaisantes sous la forme d'une fable. Elle se lance dans l'écriture pour tenter de donner un sens à ce passé « indépassable » (p.180) puisqu'elle est condamnée à vivre en 
permanence dans l'ombre du génocide ${ }^{12}$. Pourtant, elle ne trouve aucune réponse, même dans l'écriture, tant et si bien que son personnage est voué au même désir qu'elle : en finir avec la vie.

Gatore aborde avec tact le sentiment de non-existence que beaucoup de rescapés et de survivants du génocide ressentent bien après l'événement. Sandor Ferenczi a montré qu'un sujet gravement traumatisé peut être un mort-vivant, c'est-à-dire une personne qui, pour se défendre d'une surcharge émotive, se détache de son corps, protégeant le moi de toute atteinte physique importante. Comme l'explique Ferenczi, «la part morte, dématérialisée, a tendance à vouloir attirer à elle dans le non-être la partie non encore morte, en particulier dans les rêves (en particulier dans les cauchemars) » (p.55). Pour Isaro, la partie morte de son être est restée sous le lit, alors que ses parents se faisaient massacrer sous ses yeux d'enfant. Incapable d'historiciser sa propre existence et de lutter contre cette partie morte de son moi qui n'existe plus, Isaro se perd dans la culpabilité d'être vivante, puis dans la douleur et, enfin, dans la dépression.

Le lecteur averti découvre, dès le début du roman, que Gatore a adroitement glissé deux prolepses qui le renseignent adroitement sur la fin tragique de l'histoire. La première est placée dans l'écriture même d'Isaro alors qu'elle est en train de rédiger le début de son propre roman. La jeune femme, rentrée à Paris après son voyage au Rwanda, élabore une " entrée en matière » qui se lit comme suit: " "Cher inconnu, bienvenue

\footnotetext{
12 Isaro redoute de croiser les hommes dans la rue de peur de reconnaître en eux le tueur de sa famille ou encore de voir apparaître une sœur que l'on dit vivante (Gatore, p. 180).
} 
dans ce récit dont tu seras le seul survivant"»(p.12). Les termes «dont tu seras le seul survivant» annoncent en effet la mort de Niko, nous le savons déjà, mais aussi la mort d'Isaro. La jeune fille pensait qu'un retour sur sa terre natale lui donnerait toutes les réponses à sa quête intérieure. Malheureusement, la retranscription des témoignages de prisonniers a rendu son travail de reconstruction personnelle bien difficile. Commencé dans la «joie », (p.173), le projet tourne à l'obsession : tout prendre en notes, tout garder en mémoire, mais dans quel but? La deuxième prolepse, qui se trouve au douzième chapitre, anticipe l'issue fatale : "ils changeront d'avis lorsqu'ils auront entendu quelque chose tomber dans la pièce et qu'à ce bruit lourd aura succédé un silence trop long et trop parfait pour ne pas les inquiéter » (p. 175). Ne plus avoir peur, réunir leur moi fractionné (les parties morte et vivante), ne plus ressentir l'angoisse et s'abandonner au silence sans résistance pour ne plus souffrir : tel est le destin des deux protagonistes.

Comme Niko, qui est muré dans son mutisme, ce qui l'empêche de justifier ses actes, Isaro reste tout aussi silencieuse dans l'écriture de son roman et dans sa mort ${ }^{13}$. Incapable de faire face à ses souvenirs, elle va passer à l'acte ultime qui la délivrera. Gatore a bâti son roman en boucle puisque le dernier chapitre fait écho à l'incipit. En effet, on retrouve Isaro toujours «immobile», «droite» (p. 11 et 183) sur son tabouret, prête à se jeter dans le vide, "tenant fermement » la poignée en bois du poignard qu'elle tient dans ses mains (p. 184). À l'image de son personnage de roman, Niko,

13 À cet égard, Elizabeth Applegate analyse comment les deux personnages du roman sont liés par des images récurrentes et établit des connections entre leurs destins respectifs. Cette analyse nous semble parfaitement fondée puisque Niko est la création d'Isaro, qui se projette donc dans son personnage. 
qui est mort étouffé par le regard des singes, Isaro meurt asphyxiée sous le poids de son passé traumatique. Le processus créateur d'Isaro a, au début, comblé un manque en tentant de jeter des ponts sur le vide créé par le trauma du génocide. Pourtant, l'acte d'écrire n'a pas comblé son angoisse de mort et son vide psychique.

L'écriture de Gatore s'offre comme un lieu d'expérimentation dans lequel l'auteur s'autorise à dépasser — par un agencement textuel singulier - les conventions des récits fidèles au réalisme, c'est-à-dire des textes qui dépeignent la réalité génocidaire sans artifices. Le but de Gatore était simple : ne pas faire de témoignage parce que, pour lui, dire le réel fait partie de l'intime. Il l'explique dans un entretien avec Jean-Claude Perrier :

Il y a des nationalités qui pèsent plus que d'autres, dit-il. Israélien, Palestinien, ou Rwandais. On m'attend toujours làdessus. Or je ne veux en aucun cas être le énième petit Rwandais à témoigner du génocide. Je n'ai rien de plus à dire sur ce drame que ce que j'ai mis dans mon livre. Le reste appartient à l'intime.

Le silence de l'intime dans lequel s'emmurent les personnages de Gatore ressemble étrangement au silence de Gatore sur ses années passées au Rwanda, dont il préfère ne pas parler, soit qu'il a des choses à cacher comme le prétendent certaines personnes, soit qu'il était trop jeune pour se souvenir exactement de tout (voir Applegate, p. 76). En fait, Gatore n'a jamais prétendu écrire à partir de la réalité ou de son vécu. Son point de vue fictif l'emmène bien au-delà des frontières du faisable et de l'imaginable. À bien des égards, le travail de 
Gatore repousse les frontières de l'expression écrite du trauma collectif et c'est par devoir de mémoire pour ceux qui ont souffert du génocide que Gatore se devait d'aller jusqu'au bout de son projet. Comme le dit si bien Mwatha Musanji Ngalasso, «si le génocide constitue le "Mal absolu", l'écriture est une thérapie qui rétablit l'homme dans son humanité » (p. 182).

\section{Bibliographie}

APPLEGATE, Elizabeth. (2012), "Reimagining the Swallow and the Toad: Narrating Identity and Reconciliation in Postgenocide Rwanda», Research in African Literatures, vol. 43, no 1, p. 70-88.

BISANSWA, Justin. (2009), Roman africain contemporain, Paris, Honoré Champion.

COLLVVLLE, Nathalie. (2010), «Rencontre avec Gilbert Gatore», Centre régional des Lettres de Basse-Normandie, rencontre le mardi 8juin à l'Espace Senghor à Verson animée par Bernard Magnier, <http://www.crlbn.fr/2010/05/27/rencontre-avec-gilbertgatore/>.

Coquio, Catherine. (2010), "Poétiser l'enfant tueur», dans Déborah Lévy-Bertherat et Pierre Schoentjes (dir.), "J'ai tué ». Violence guerrière et fiction, Genève, Droz, p. 231-265.

FERENCZI, Sandor. (2006 [1932]), «Penser le corps, c'est comme l'hystérie », dans Le Traumatisme, Paris, Petite bibliothèque Payot, p. 61-68.

-. (2006 [1930]), «De la construction analytique des mécanismes psychiques », dans Le Traumatisme, p. 53-55. 
FREUD, Sigmund. (1968 [1920]), «Au-delà du principe de plaisir », traduit de l'allemand par S. Jankélévitch, dans Essais de psychanalyse, Paris, Payot, p. 7-82, $<$ http://classiques.uqac.ca/classiques/freud_sigmund/essai s_de_psychanalyse/Essai_1_au_dela/au_dela_prin_plaisir.ht $\underline{\mathrm{ml}}>$.

-. (1968 [1915]), « Considérations actuelles sur la guerre et sur la mort», traduit de l'allemand par S. Jankélévitch, dans Essais de psychanalyse, Paris, Payot, p. 235-267, $<$ http://classiques.uqac.ca//classiques/freud_sigmund/essa is_de_psychanalyse/Essai_4_considerations/considerations. html>.

Gatore, Gilbert. (2008), Le Passé devant soi, Paris, Phébus, coll. «10-18».

-. (2011), "Rencontre avec Gilbert Gatore, auteur du roman Le Passé devant soi », Institut français de Rome, vidéo, 54’02, $<$ http://www.dailymotion.com/video/xhz1r5_rencontreavec-gilbert-gatore-auteur-du-roman-le-passe-devantsoi_creation>.

-. (2012). The Past Ahead, traduit par Marjolijn de Jager, Bloomington, University Press, coll. « Global African Voices ».

GENETTE, Gérard. (1972), Figures III, Paris, Seuil.

GODARD, Marie-Odile. (2003), Rêves et traumatismes ou la longue nuit des rescapés, Ramonville Saint-Agen, Érès.

Hiтcнсотт, Nicki. (2013), «Between Remembering and Forgetting: (In)Visible Rwanda in Gilbert Gatore's Le Passé devant soi ", Research in African Literatures, vol. 44, n으. p. 76-90. 
ISER, Wolfgang. (1985), L'Acte de lecture. Théorie de l'effet esthétique, traduction de l'allemand par Evelyne Sznycer, Bruxelles, Mardaga.

MujaWAyo, Esther et Souâd Belhaddad. (2006), La Fleur de Stéphanie, Paris, Flammarion.

MukASONGA, Scholastique. (2012), Notre-Dame du Nil, Paris, Gallimard, coll. « Continents noirs ».

-. (2010), L'Iguifou. Nouvelles rwandaises, Paris, Gallimard, coll. «Continents noirs».

-. (2008), La Femme aux pieds nus, Paris, Gallimard, coll. «Folio».

-. (2006), Inyenzi ou les Cafards, Paris, Gallimard, coll. «Continents noirs».

NGALASSO-MwATHA, Musanji. (2008), « Le trauma dans la littérature africaine », dans Peter Kuon (dir.), Trauma et texte, New York, Peter Lang, p. 161-183.

Perrier, Jean-Claude. (2008), «Gilbert Gatore, de l'enfer du Rwanda à HEC », Le Figaro, <http://www.lefigaro.fr/livres/2008/07/04/0300520080704ARTFIG00309-gilbert-gatorede-l-enfer-durwanda-a-hec.php>.

SEHEne, Benjamin. (2205), Le Feu de la soutane, Paris, L’Esprit frappeur.

Semunjanga, Josias. (2008), Le Génocide, sujet de Fiction? Analyse des récits du massacre des Tutsi dans la littérature africaine, Québec, Nota bene. 


\title{
Résumé
}

Cet article se propose d'examiner le dispositif fictionnel du livre du Rwandais Gilbert Gatore intitulé Le Passé devant soi (2008), qui s'inscrit dans un projet de réflexion sur le génocide des Tutsi en 1994 en s'écartant délibérément du réalisme ordinaire que l'on retrouve dans la plupart des textes publiés sur ce sujet poignant. En effet, Gatore offre une fable dans laquelle le destin de Niko le paria reflète étrangement celui de la belle Isaro, deux victimes, l'une entraînée par la folie meurtrière du mal, l'autre dépossédée de son identité. C'est à travers ces deux personnages solitaires qui résistent autant qu'ils le peuvent à la peur d'affronter une vérité qui dérange que Gatore tente de répondre à la question introduite en épigraphe à son ouvrage : "Que vaut-il mieux faire lorsque, sans aucun doute, il est trop tard?»

\begin{abstract}
This article details and examines the work of the intertwined narrative structure of Gilbert Gatore's book, Le Passé devant soi (2008), a fictional novel which laid the evidentiary basis for the reader to reflect on the Tutsi Genocide of 1994 in a manner that distinguishes it from traditional testimonies and classical autobiographical texts. Gatore presents a fable in which the destiny of Niko, a mute social outcast, reflects strangely on the fate of the beautiful Isaro, two victims: one who unwillingly becomes manipulated by the chaotic tidal waves of evil, the other stricken from her own identity. It is through these two protagonists, who resist as much as they can to the horror of confronting the truth that troubles them, that Gatore attempts to answer his self-induced rhetorical question in an epigraph to his text: "What better is there to be done when there's no doubt whatsoever that it's too late?"
\end{abstract}

